Supporting information for

\title{
Modulation of the Sensitive Temperature Range of Fluorescent Molecular Thermometers Based on Thermo-Responsive Polymers
}

\author{
Seiichi Uchiyama, ${ }^{\dagger}{ }^{\dagger}$ Yuriko Matsumura, ${ }^{\dagger}$ A. Prasanna de Silva, ${ }^{*}$ Kaoru Iwai ${ }^{\dagger}$ \\ †Department of Chemistry, Faculty of Science, Nara Women's University, Kitauoya-Nishimachi, Nara 630-8506, \\ Japan \\ ${ }^{\dagger}$ School of Chemistry, Queen's University, Belfast, Northern Ireland, Belfast BT9 5AG, UK
}
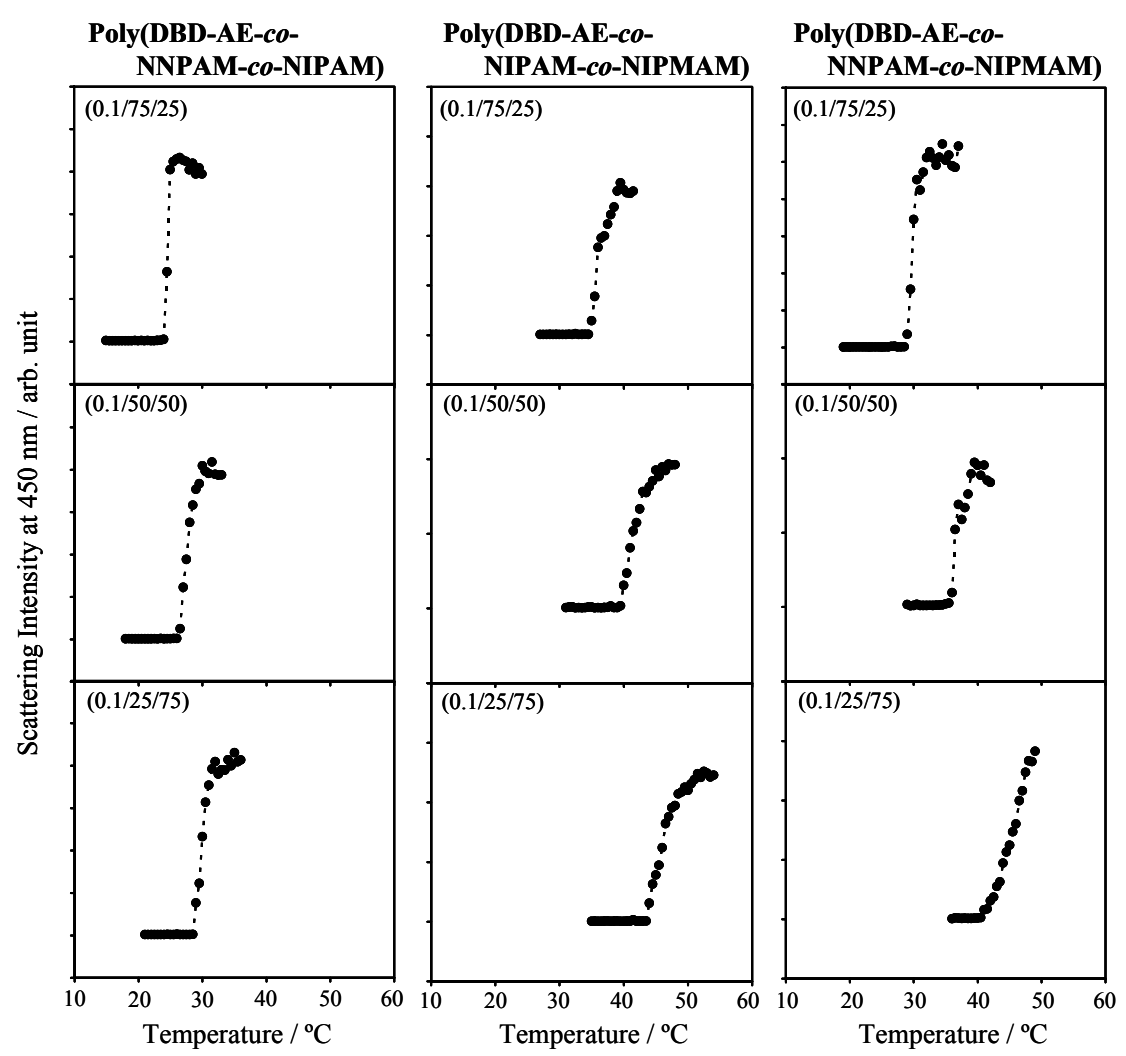

Figure S1. Relationships between the temperatures and the scattering intensities of poly(DBD-AE-co-NNPAM-co-NIPAM), poly(DBD-AE-co-NIPAM-co-NIPMAM), and poly(DBD-AE-co-NNPAM-co-NIPMAM). The excitation wavelength was $444 \mathrm{~nm}$. 


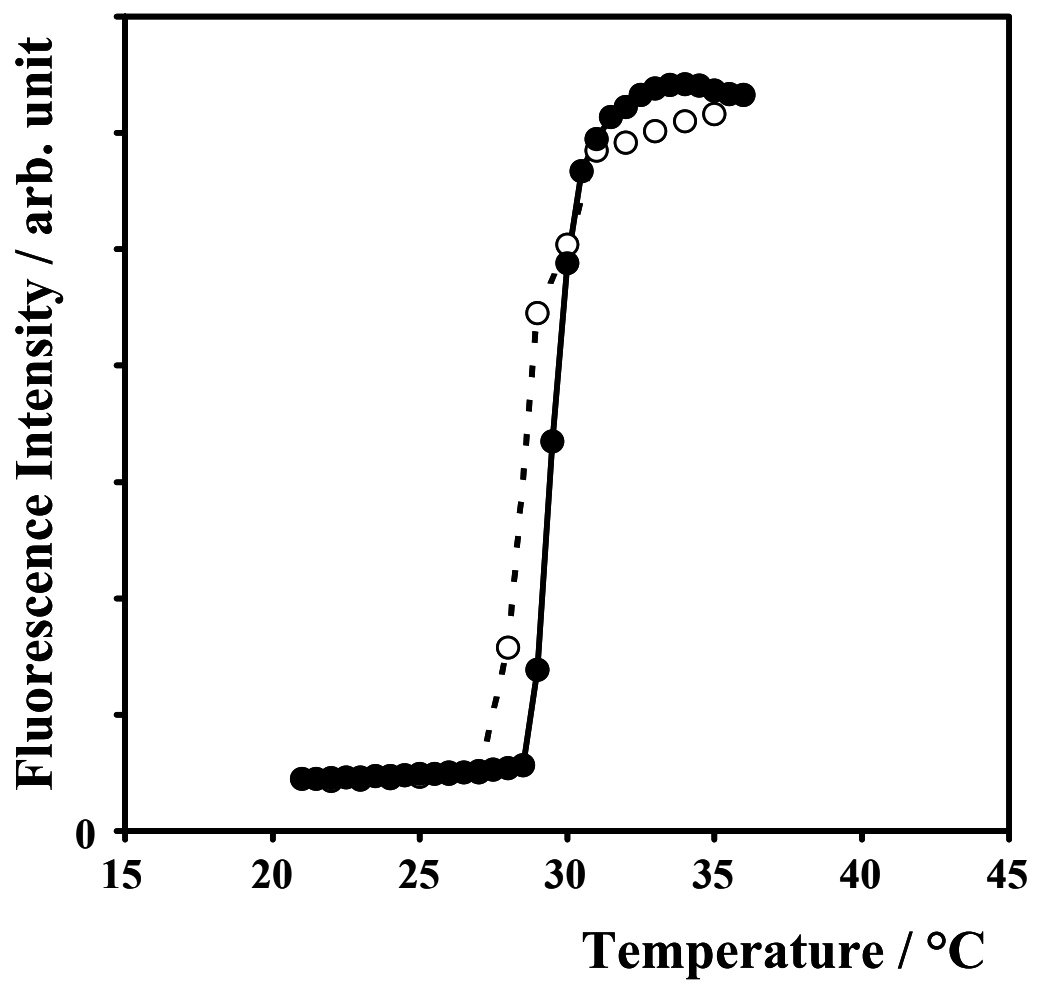

Figure S2. Hysteresis of poly(DBD-AE-co-NNPAM-co-NIPAM) $(0.1 / 25 / 75)$ as a representative; relationships between the temperatures and the normalized fluorescence intensities at maximum emission wavelengths with heating ( $-\bullet)$ and cooling $(\boldsymbol{\cdots} \circ \cdot \boldsymbol{*})$ in aqueous solution $(0.01 \mathrm{w} / \mathrm{v} \%)$. Fluorescence intensities were normalized by using the fluorescence intensity at $26^{\circ} \mathrm{C}\left(\mathrm{T}_{\text {low }}\right)$. 
Table S1. Sensitivities and reproducibilities of the developed fluorescent molecular thermometers ${ }^{a}$

\begin{tabular}{|c|c|c|c|c|c|}
\hline copolymer & $\begin{array}{l}\mathrm{T}_{\text {low }}{ }^{b} \\
\left({ }^{\circ} \mathrm{C}\right)\end{array}$ & $\begin{array}{c}\mathrm{T}_{\text {high }}{ }^{b} \\
\left({ }^{\circ} \mathrm{C}\right)\end{array}$ & $\begin{array}{l}\Delta \mathrm{T}^{b} \\
\left({ }^{\circ} \mathrm{C}\right)\end{array}$ & $\mathrm{FE}^{c}$ & $\begin{array}{c}\mathrm{FE} / \Delta \mathrm{T} \\
\left({ }^{\circ} \mathrm{C}^{-1}\right)\end{array}$ \\
\hline \multicolumn{6}{|c|}{ poly(DBD-AE-co-NNPAM-co-NIPAM) } \\
\hline$(0.10 / 75 / 25)$ & 22.5 & 26.5 & 4.0 & 13.4 & 3.4 \\
\hline$(0.10 / 50 / 50)$ & 26.0 & 28.5 & 2.5 & 11.6 & 4.6 \\
\hline$(0.10 / 25 / 75)$ & 28.0 & 31.5 & 3.5 & 11.4 & 3.3 \\
\hline \multicolumn{6}{|c|}{ poly(DBD-AE-co-NIPAM-co-NIPMAM) } \\
\hline$(0.10 / 75 / 25)$ & 34.5 & 38.5 & 4.0 & 11.6 & 2.9 \\
\hline$(0.10 / 50 / 50)$ & 39.0 & 43.5 & 4.5 & 9.29 & 2.1 \\
\hline$(0.10 / 25 / 75)$ & 43.5 & 48.0 & 4.5 & 7.39 & 1.6 \\
\hline \multicolumn{6}{|c|}{ poly(DBD-AE-co-NIPAM-co-NIPMAM) } \\
\hline$(0.10 / 75 / 25)$ & 28.0 & 33.0 & 5.0 & 14.5 & 2.9 \\
\hline$(0.10 / 50 / 50)$ & 34.0 & 39.5 & 5.5 & 10.1 & 1.8 \\
\hline$(0.10 / 25 / 75)$ & 40.0 & 46.5 & 6.5 & 8.04 & 1.2 \\
\hline c.f. poly(DBD-AE-co-NNPAM) & 19.0 & 23.0 & 4.0 & 14.9 & 3.7 \\
\hline c.f. poly(DBD-AE-co-NIPAM) & 31.5 & 36.0 & 4.5 & 11.4 & 2.5 \\
\hline c.f. poly(DBD-AE-co-NIPMAM) & 46.0 & 53.5 & 7.5 & 6.75 & 0.90 \\
\hline
\end{tabular}

